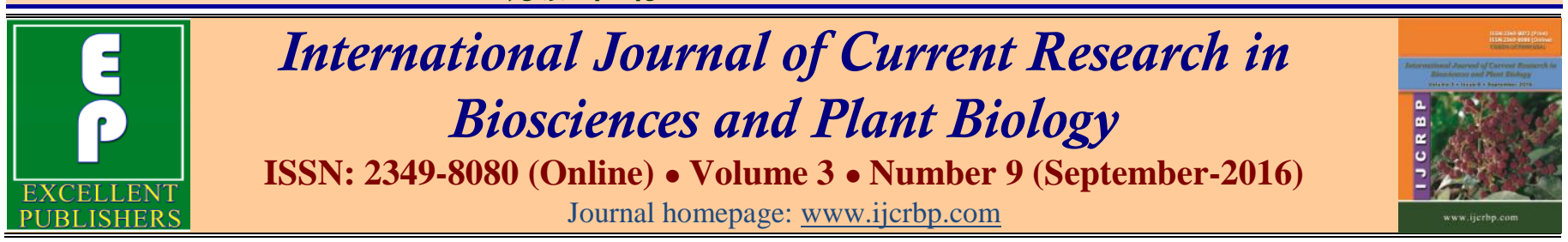

Original Research Article

doi: http://dx.doi.org/10.20546/ijcrbp.2016.309.005

\title{
Molecular Cloning and Sequence Analysis of a Novel Geranylgeranyl Diphosphate Synthase Gene (GbGGPPS2) from Ginkgo biloba
}

\author{
Xiaoqiang Li, Weiwei Zhang and Feng Xu* \\ College of Horticulture and Gardening, Yangtze University, Jingzhou 434025, China \\ *Corresponding author.
}

\section{A b stract}

Geranylgeranyl diphosphate synthase (GGPPS), a key enzyme in the biosynthesis of ginkgolide, catalyzes the formation of geranylgeranyl diphosphate (GGPP) which is a biosynthetic precursor for ginkgolide. In this study, a novel full-length cDNA encoding GGPPS was isolated, designated as GbGGPPS2 (GenBank accession no. KX756450), from Ginkgo biloba. The full-length cDNA of GbGGPPS2 was $1568 \mathrm{bp}$ and contained a $1149 \mathrm{bp}$ open reading frame (ORF). The molecular weight and isoelectric point of the GbGGPPS2 protein was $129.9 \mathrm{kDa}$ and 5.01, respectively. Multiple alignments analysis revealed that GbGGPPS2 showed extensive homology with GGPPSs from other species and possessed five conservative domains, indicating that the GbGGPPS2 belonged to GGPPS gene family. Phylogenetic tree analysis showed that GbGGPPS2 shared the same ancestor in evolution with other GGPPSs and had a further relationship with other gymnosperm species.
\end{abstract}

\section{Introduction}

Ginkgo biloba is one of the most popular medicinal plants. It has been existing over 200 million years on the earth (He et al., 2009). The ginkgo leaf contains many active ingredients including ginkgolides, flavonol and flavone glycosides, diterpene lactones, sesquiterpenes, iron-based superoxide dismutase, phydroxybenzoic acid, ascorbic acid, and catechin (Jacobs and Browner, 2000). Ginkgolides, belonging to diterpenes, were first isolated by Furukawa from the root bark (Sabater-Jara et al., 2013). It has beneficial effect on cardiovascular and neurological systems (Lin et al., 1994). Further, the efficacy of reducing blood pressure and attack of migraine frequency has been proved (Usai and Bussone, 2011). Therefore, improving the quality and quantity of ginkgolide can bring tremendous medical effects and commercial value.
Terpenoids, a class of compound, are composited with several isoprene (Tholl, 2006). Isopentenyl diphosphate (IPP) is a universal biosynthetic precursor of isoprenoids. In plants, there are two pathway to synthesise IPP: mevalonate (MVA) pathway in cytoplasm and 2-Cmethyl-D-erythritol-4-phosphate (MEP) pathway in plastids (Yu and Utsumi, 2009). In MVA pathway, acetyl-CoA finally synthesize IPP through a series of enzymatic reaction (Newman and Chappell, 1999); In MEP pathway, the reactant are both pyruvate and glyceraldehyde-3-phosphate (GA3P) and the final resultant is IPP (Cordoba et al., 2009). Geranylgeranyl diphosphate (GGPP), a key common diterpene precursor, is biosynthesized by geranylgeranyl diphosphate synthase (GGPPS) in the plastids (Fig 1; Dewick, 2002; Liao et al., 2004). GGPPS is a prenyltransferase that catalyzes the electrophilic coupling of farnesyl diphosphate (FPP) and IPP (Walker and Croteau, 2001). GGPPS protein contains five conservative domains: 
domain I, II, III, IV and V. Meanwhile, two of these conservative domains are the first aspartate-rich motifs (FARM) and the second aspartate-rich motif (SARM) respectively (Engprasert et al., 2004).

\section{MVA pathway}

\section{MEP pathway}

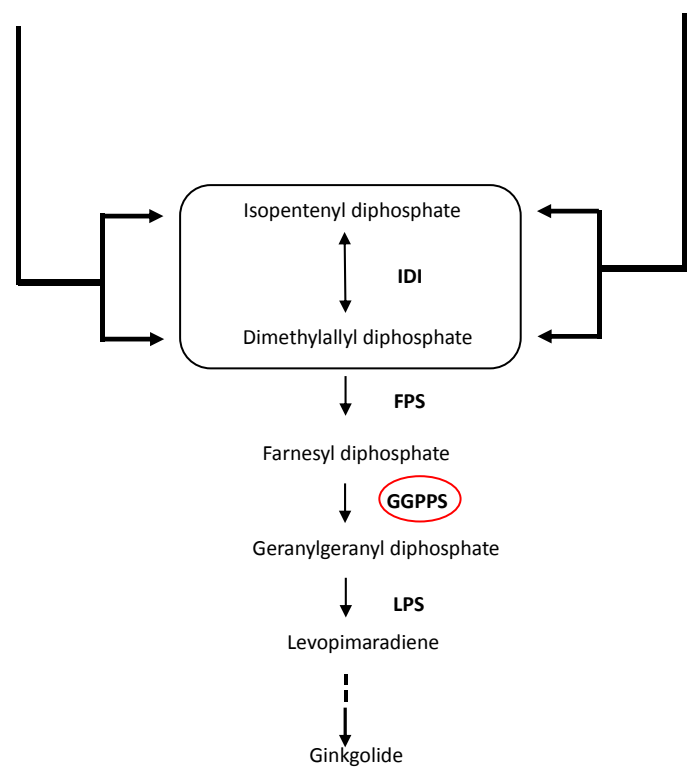

Fig. 1: Ginkgolides biosynthesis pathways in Ginkgo biloba.

In our previous report, transcriptome analysis of $G$. biloba was performed by us through using Illumina HiSeq ${ }^{\mathrm{TM}} 2500$ sequencing platform. Based on the transcriptome data, we cloned a novel GGPPS gene from $G$. biloba and analyzed the structure of the sequence, aiming to provide the gene resource for increase the content of ginkgolide in G. biloba using genetic engineering.

\section{Materials and methods}

\section{Plant material and reagents}

The 15-year-old grafts of G. biloba were grown in the Botanical Garden of Yangtze University, China. The leaves of G. biloba were collected and preserved in a $-80^{\circ} \mathrm{C}$ refrigerator immediately. Both of Primer synthesis and DNA sequencing were performed by Shanghai Sangon Biotechnology Company, in China.

\section{Cloning the full-length cDNA of GbGGPPS2}

Total RNA of G. biloba was isolated from young leaves using the CTAB method described by Cai et al. (2007). The first-strand cDNA was synthesized according to the instruction of PrimeScript ${ }^{\mathrm{TM}}$ 1st Strand cDNA Synthesis
Kit (TaKaRa, Dalian, China). Using the software of DNAMAN V6, the specific primers GbGGPPS-U (5'-TAGGAAGGAGGACAGAACAGTATC-3') and GbGGPPS-D (5'-TTGATATCTAATTCTGTCTGTGAGC$3^{\prime}$ ) were designed based on the GGPPS unigene sequence of ginkgo transcriptome data. GbGGPPS 2 cDNA was amplified under the following conditions: perdenaturation at $94^{\circ} \mathrm{C}$ or $3 \mathrm{~min}$; denaturation at $94^{\circ} \mathrm{C}$ for $30 \mathrm{sec}$; anneal at $51^{\circ} \mathrm{C}$ for $30 \mathrm{sec}$; extension at $72^{\circ} \mathrm{C}$ for $90 \mathrm{sec}$. By 35 cycles, extend at $72^{\circ} \mathrm{C}$ for $10 \mathrm{~min}$. The amplified product was purified, ligated into pMD18-T vector, and introduced into Escherichia coli strain DH5 $\alpha$ followed by sequencing.

\section{Bioinformatics and molecular evolution analysis}

The sequence was analyzed by the bioinformatics software on websites (http://www.xpasy.org and http://www.ncbi.nlm.nih.gov), Plant GGPPS protein sequences were retrieved from NCBI GenBank. Sequence alignment was performed using ClustalX 2.0 and phylogenetic tree was constructed by neighborjoining method using MEGA 6.0. A bootstrap statistical analysis was carried out with 1000 replicates.

\section{Results and discussion}

\section{Cloning and sequence analysis of GbGGPPS2}

The cDNA of GbGGPPS2 (GenBank accession no. KX756450) was cloned by a pair of specific primers based on the GbGGPPS2 unigene of transcriptome data. The full-length of GbGGPPS2 was 1568 bp and contained a 1149 bp opening read frame (ORF) which encoded a peptide of 383 amino acids (Fig. 2). In NCBI database, the nucleotide sequence of GbGGPPS2 revealed high similarity with the entire coding region of GGPPS genes in Populus euphratica (81\%), Populus trichocarpa (81\%), Lycium chinense (79\%), Solanum pennellii (76\%). Thus, the GbGGPPS 2 might be a member of GGPPS2 gene family.

\section{Characterization of GbGGPS2 protein}

The calculated isoelectric point (pI) and molecular weight of $G b G G P P S 2$ were predicted to be 5.01 and $129.9 \mathrm{kDa}$, respectively (http://cn.expasy.org/tools/ protparam.html). Multi-alignment of GbGGPPS2 with other plant GGPPSs showed high similarity (Fig. 3). The amino acid sequence of the GbGGPPS2 was highly similar to previously reported TcGGPPS of Taxus canadensis (Hefner and Croteau, 1998). 


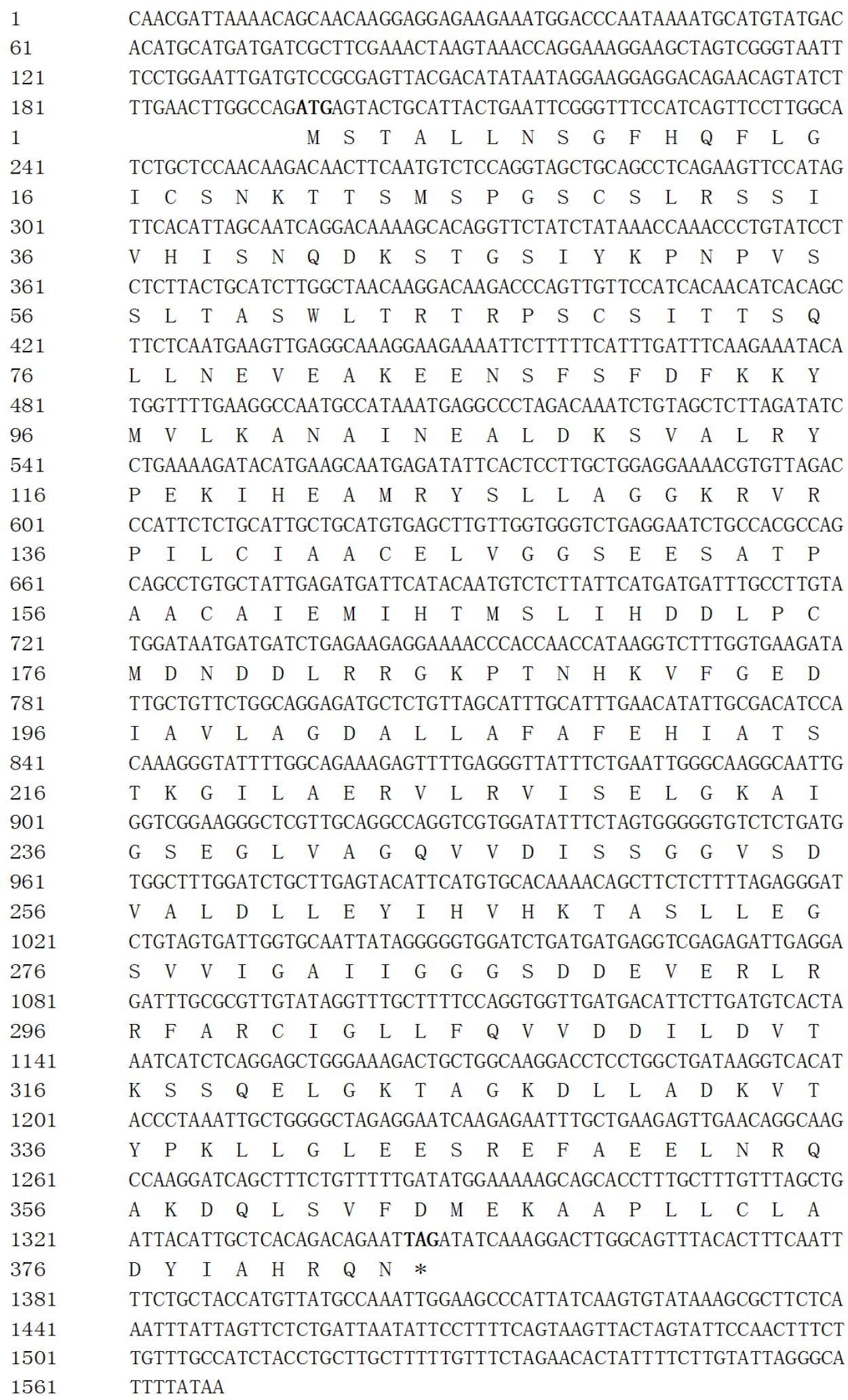

Fig. 2: Nucleotide sequence and deduced amino acid sequence of GbGGPP2.

Amino acid sequence alignment of GbGGPPS2 and other plant GGPPS showed that GbGGPPS2 exhibited high similarity to GGPPS proteins from other plants.
The deduced GbGGPPS2 protein sequence showed $76 \%, 74 \%, 76 \%, 76 \%, 77 \%, 75 \%, 77 \%$ and $75 \%$ identities to the counterparts of PaGGPPS6 from Picea 
abies, GbGGPPS1 from G. biloba, LaGGPPS from Lepidium apetalum, CrGGPPS from Catharanthus roseus, EuGGPPS from Eucommia ulmoides, CmGGPPS from Cucumis melo, CaGGPPS from Corylus avellana, TkGGPPS1 from Taraxacum koksaghyz. Homology analysis with GGPPSs from other plants revealed that GbGGPPS2 contained five conserved structural domains. Meanwhile, The second and the fifth domain was the first and the second aspartate-rich motifs respectively, which were important for the catalytic activity of GGPPS (Ashby and Edwards, 1990).
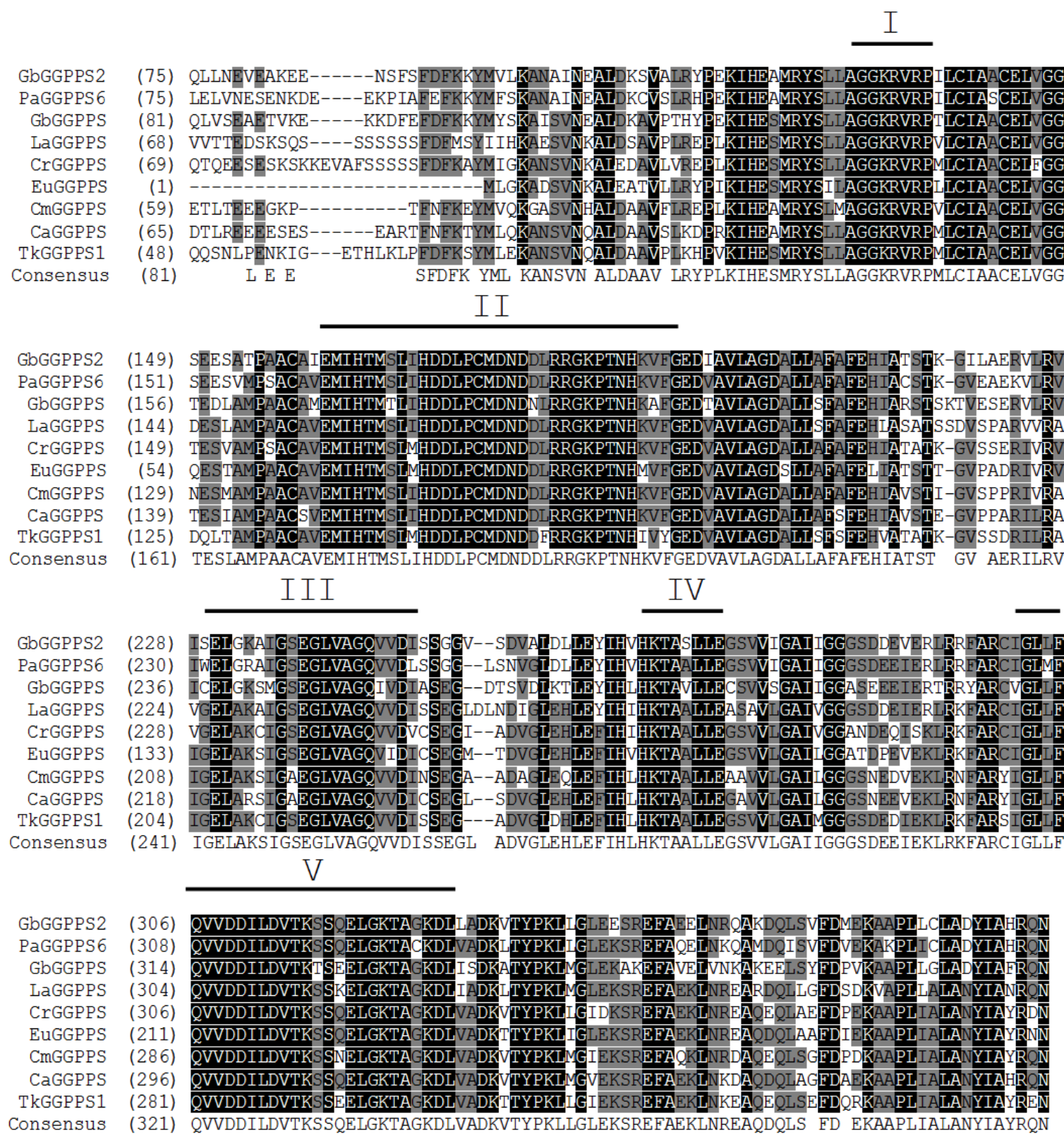

Fig. 3: Multiple alignments of the deduced amino acid sequences of GbGGPPS2 with other GGPPS proteins. The accession numbers of GGPPS proteins and translation of their names are shown as follows, GbGGPPS2: Ginkgo biloba (KX756450); PaGGPP6: Picea abies (ACA21462.1); GbGGPPS: Ginkgo biloba (AAQ72786.1); LaGGPPS: Lepidium apetalum (AKQ24577.1); CrGGPPS: Catharanthus roseus (AE153622.1); EuGGPPS: Eucommia ulmoides (AGJ03661.1); CmGGPPS: Cucumis melo (AGN48013.1); CaGGPPS: Corylus avellana ABW06960.1; TkGGPPS1: Taraxacum koksaghyz (AMB19719.1). The completely identical amino acids are indicated with white foreground and black background. Shaded in gray are conservative sequences. The five conserved motifs were marked. 


\section{Molecular evolution analysis}

To investigate the evolutionary relationships among GGPPS proteins including GbGGPPS2, a phylogenetic tree was constructed based on the deduced amino acid sequences of predicted GbGGPPS2 and GGPPS proteins from other plant species (Fig. 4). The GGPPSs of the analyzed plants were divided into two main groups: angiosperms and gymnosperms. Fig. 4 shows that the GbGGPPS 2 belongs to gymnosperm and has the closest relationship with PaGGPPS6. As expected, GbGGPPS2 belonged to gymnospermous GGPPS family.

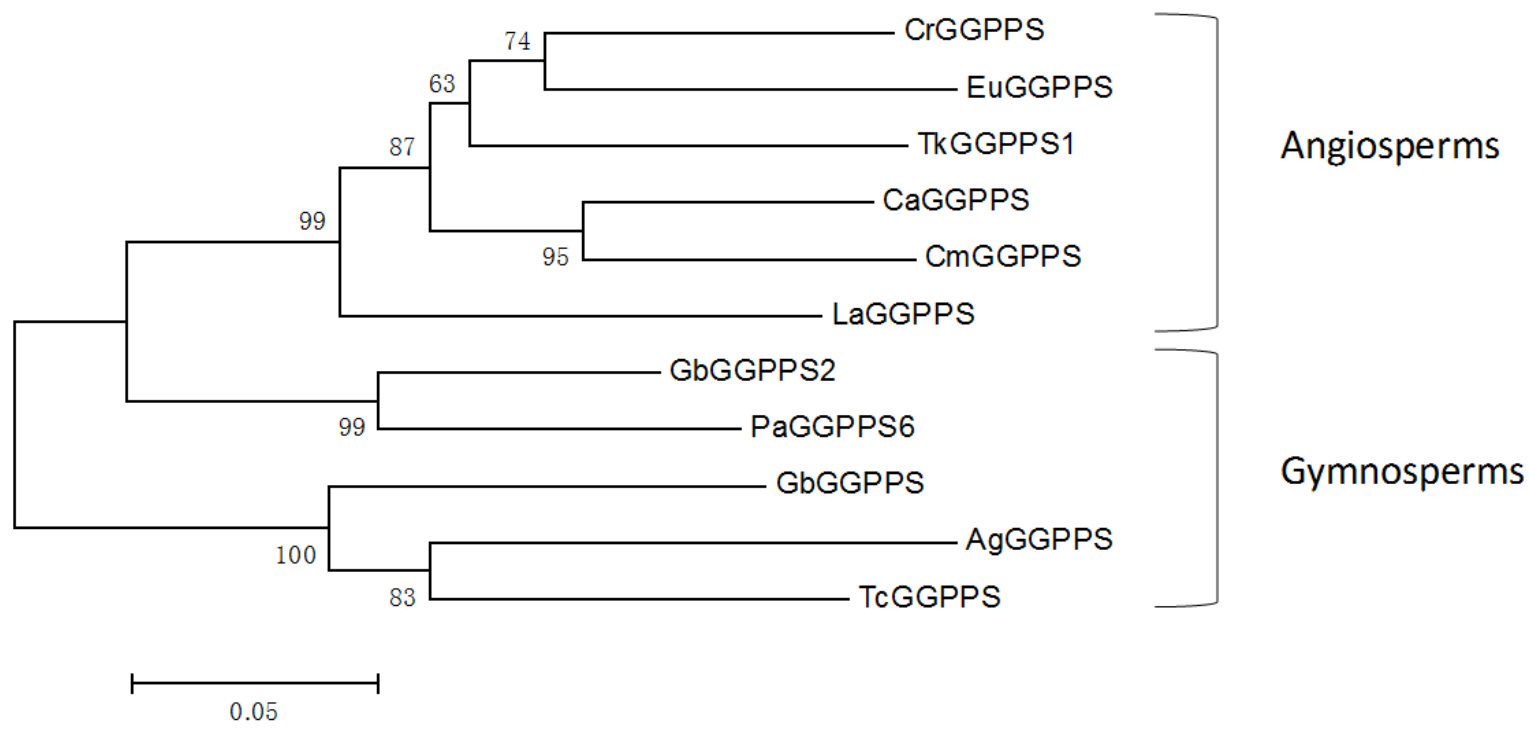

Fig. 4: Phylogenetic tree of the sequences of GbGGPPS2 and other plants GGPPS protein. Bootstrap value are expressed in percentages and placed at the nodes in the tree. The GenBank accession numbers of the GGPPS proteins and translation of their names are shown, CrGGPPS: Catharanthus roseus (AE153622.1); EuGGPPS: Eucommia ulmoides (AGJ03661.1); TkGGPPS1: Taraxacum koksaghyz (AMB19719.1); CaGGPPS: Corylus avellana (ABW06960.1); CmGGPPS: Cucumis melo (AGN48013.1); LaGGPPS: Lepidium apetalum (AKQ24577.1); GbGGPPS2: Ginkgo biloba (KX756450); PaGGPP6: Picea abies (ACA21462.1); GbGGPPS: Ginkgo biloba (AAQ72786.1); AgGGPPS: Abies grandis (AAL17614.2); TcGGPPS: Taxus canadensis (AAD16018.1).

\section{Conclusion}

In this study, we have successfully isolated and characterized of the gene GbGGPPS2 encoding GGPPS involved in the biosynthesis of diterpenes in G. biloba. The multiple sequences alignment by using bioinformatics analysis software indicated that GbGGPPS2 had high identity with other GGPPS genes isolated from other plants. The phylogenetic analysis also showed that GbGGPPS2 might keep a strong conservation during the molecular evolution. The cloning and characterization of GbGGPPS2 will provide a theoretical basis for enhancement ginkgolide in G. biloba through genetic engineering.

\section{Conflict of interest statement}

Authors declare that they have no conflict of interest.

\section{Acknowledgement}

This work was supported by National Natural Science Foundation of China (31370680) and the Natural Science Foundation of Hubei Province (No. 2013CFA039).

\section{References}

Ashby, M. N., Edwards, P. A., 1990. Elucidation of the deficiency in two yeast coenzyme $Q$ mutants. Characterization of the structural gene encoding hexaprenyl pyrophosphate synthetase. J. Biol. Chem. 265(22), 13157-13164.

Cai, R., Xu, F., Chen, L. J., Cheng, S. Y., 2007. Modification of total RNA isolation method from different Ginkgo biloba organs. Biotechnol. 17(4), 38-41.

Cordoba, E., Salmi, M., León, P., 2009. Unravelling the regulatory mechanisms that modulate the MEP pathway in higher plants. J. Exp. Bot. 60(10), 2933-2943. 
Dewick, P. M., 2002. The biosynthesis of C 5-C 25 terpenoid compounds. Nat. Prod. Rep. 19(2), 181-222.

Engprasert, S., Taura, F., Kawamukai, M., Shoyama, Y., 2004. Molecular cloning and functional expression of geranylgeranyl pyrophosphate synthase from Coleus forskohlii Briq. BMC Plant biol. 4(1), 1.

He, X., Huang, W., Chen, W., Dong, T., Liu, C., Chen, Z., Xu, S., Ruan, Y., 2009. Changes of main secondary metabolites in leaves of Ginkgo biloba in response to ozone fumigation. J. Environ. Sci. 21(2), 199-203.

Hefner, J., Ketchum, R. E., Croteau, R., 1998. Cloning and functional expression of a cDNA encoding geranylgeranyl diphosphate synthase from Taxus canadensis and assessment of the role of this prenyltransferase in cells induced for taxol production. Arch. Biochem. Biophys. 360(1), 62-74.

Jacobs, B. P., Browner, W. S., 2000. Ginkgo biloba: A living fossil. Am. J. Med. 108(4), 341-342.

Liao, Z., Chen, M., Gong, Y., Guo, L., Tan, Q., Feng, X., Tang, K., 2004. A new geranylgeranyl diphosphate synthase gene from Ginkgo biloba, which intermediates the biosynthesis of the key precursor for ginkgolides. DNA Sequence. 15(2), 153-158.
Lin, L., Gu, H., Gao, J., Wu, J., Zhang, H., Tang, G., Zhang, Z., 1994. Protective effect of ginkgotins on glutamate neurotoxicity in vitro. J. Nanjing Univ. 31(3), 514-518.

Newman, J. D., Chappell, J., 1999. Isoprenoid biosynthesis in plants: carbon partitioning within the cytoplasmic pathway. Crit. Rev. Biochem. Mol. 34(2), 95-106.

Sabater-Jara, A. B., Souliman-Youssef, S., Novo-Uzal, E., Almagro, L., Belchí-Navarro, S., Pedreño, M. A., 2013. Biotechnological approaches to enhance the biosynthesis of ginkgolides and bilobalide in Ginkgo biloba. Phytochem. Rev. 12(1), 191-205.

Tholl, D., 2006. Terpene synthases and the regulation, diversity and biological roles of terpene metabolism. Curr. Opin. Plant biol. 9(3), 297-304.

Usai, S., Grazzi, L., Bussone, G., 2011. Gingkolide B as migraine preventive treatment in young age: results at 1year follow-up. Neurol. Sci. 32(1), 197-199.

Walker, K., Croteau, R., 2001. Taxol biosynthetic genes. Phytochemistry. 58(1), 1-7.

Yu, F., Utsumi, R., 2009. Diversity, regulation, and genetic manipulation of plant mono-and sesquiterpenoid biosynthesis. Cell. Mol. Life Sci. 66(18), 3043-3052.

\section{How to cite this article:}

Li, X., Zhang, W., Xu, F., 2016. Molecular cloning and sequence analysis of a novel geranylgeranyl diphosphate synthase gene (GbGGPPS2) from Ginkgo biloba. Int. J. Curr. Res. Biosci. Plant Biol. 3(9), 40-45. doi: http://dx.doi.org/10.20546/ijcrbp.2016.309.005 